OPEN ACCESS

Edited by:

Eddy J. Davelaar,

Birkbeck, University of London, United Kingdom

Reviewed by:

Harold H. Greene,

University of Detroit Mercy,

United States

Thomas Jacobsen,

Helmut Schmidt University, Germany

${ }^{*}$ Correspondence:

Hiroatsu Fukuda

fukuda@kitakyu-u.ac.jp

Specialty section:

This article was submitted to

Cognitive Science,

a section of the journal

Frontiers in Psychology

Received: 14 August 2021 Accepted: 16 December 2021

Published: 27 January 2022

Citation:

Dai A, Wang J, Yu J and Fukuda $H$ (2022) The Influence of Viewing Time and Color on Architectural Aesthetic Judgment.

Front. Psychol. 12:752996. doi: 10.3389/fpsyg.2021.752996

\section{The Influence of Viewing Time and Color on Architectural Aesthetic Judgment}

\author{
Anbang Dai ${ }^{1}$, Junru Wang ${ }^{1}$, Jie Yu${ }^{2}$ and Hiroatsu Fukuda ${ }^{1 *}$ \\ ${ }^{1}$ Department of Architecture, The University of Kitakyushu, Kitakyushu, Japan, ${ }^{2}$ Department of Neurology and Brain Medical \\ Center, First Affiliated Hospital, School of Medicine, Zhejiang University, Hangzhou, China
}

Understanding the factors influencing the aesthetic experience of architectures is an important topic in empirical aesthetics. In this study, we examined the effect of three architectural factors, i.e., ceiling height, openness, and contour, on viewers' aesthetic appreciation through a series of experiments. In previous studies on architectural aesthetics, participants were usually asked to view an image of an architectural space for a few seconds. The long viewing time allows them to focus on different parts of the architecture and then make an aesthetic judgment. The long viewing time, however, also makes it difficult to obtain viewers' aesthetic scores for a large number of architectural spaces in a short period. In this study, we shortened the visual presentation time to $200 \mathrm{~ms}$, which allowed the viewers to have only one fixation on the image, and asked the viewers to make an aesthetic judgment. It was found that the experiment with a 200ms viewing time could establish how the three architectural factors influenced aesthetic judgment as well as previous experiments with a 3,000-ms viewing time, suggesting that aesthetic judgment could be made within one fixation. Additionally, we investigated the impact of color on architectural aesthetic judgment by presenting grayscale images. We found that the three architectural factors influenced aesthetic judgment in similar ways for both color and grayscale images. In summary, we found that color was not a main factor modulating viewers' architectural aesthetic judgments, and we also presented a way to quickly obtain aesthetic scores for architectural spaces.

\section{Keywords: neuroaesthetic, visual perception, cognition, architecture, viewing time}

\section{INTRODUCTION}

What factors drive our aesthetic experience is a topic that receives a considerable amount of attention. The field of empirical aesthetics emphasizes that aesthetic experience should be empirically studied and experimentally validated. Using the empirical aesthetics approach, it has been shown that the relationship between complexity and novelty, and aesthetic appreciation has an inverted $U$ shape - People generally prefer an intermediate level of complexity and novelty (Berlyne, 1973). Empirical aesthetics studies have revealed that the evaluation or production of beauty, ugliness, prettiness, harmony, elegance, shapeliness or charm is governed by a host of factors, such as stimulus symmetry, complexity, novelty, familiarity, artistic style, appeal to social status, and individual preferences (Jacobsen, 2006). 
Here, we focus on aesthetic judgment of architectural inner space. Understanding people's preference for architectural features allows architects to better design structures that satisfy the aesthetic needs of the public. A good architectural design will potentially enhance the user's comfort, cognition and creativity (Sternberg and Wilson, 2006). Vegetation, stylistic uniformity, homogeneity, and symmetry can influence aesthetic experience and judgment (Jacobsen, 2006; Weber et al., 2008). Contour is also a very important feature of architectural space. A series of studies have shown that most people believe that curvilinear contours are more beautiful than rectilinear contours (Leder and Carbon, 2005; Bar and Neta, 2006; Dazkir and Read, 2011; Vartanian et al., 2013). Ceiling height and openness also affect people's cognition and emotion in an architectural space (Franz et al., 2005; Meyers-Levy et al., 2007): Under a high ceiling, people tend to have more positive emotions, such as "happy," "comfortable" and "fun." Similarly, they tend to experience more positive emotions in spacious architectural spaces than in tight architectural spaces (Stamps, 2010, 2011).

Previous studies have established that ceiling height, openness and contour can affect aesthetic judgment of architectural space. In these studies, however, participants were usually asked to view an image of an architectural space for a few seconds. The long viewing time allowed participants to focus on different parts of the architectural space before making an aesthetic judgment. The long viewing time, however, also makes it difficult to obtain viewers' aesthetic judgment for a large number of architectural spaces in a short period. In this study, we shortened the viewing time to $200 \mathrm{~ms}$, which allowed the viewers to have only one fixation on the architectural space. Furthermore, we tested whether color, a salient visual feature, can influence how architectural features modulated aesthetic experience.

\section{MATERIALS AND METHODS}

\section{Participants}

The participants in this study were students at Zhejiang University. They were all right-handed and had no visual impairment or colorblindness. All participants had normal or corrected-to-normal vision and no history of psychosis or neuropathy. The experimental procedure was approved by the Research Ethics Committee of Zhejiang University School of Medicine. All participants signed written informed consent forms before the experiment and received a monetary reward after completing the experiment. A total of 42 participants were recruited. For the condition presenting colorful images, 21 participants were recruited, including 15 males (aged $23.13 \pm 2.00$ years) and 6 females (aged $23.33 \pm 2.25$ years). For the condition presenting grayscale images, 21 participants were recruited, including 9 males (aged $22.89 \pm 1.90$ years) and 12 females (aged $22.25 \pm 1.60$ years).

\section{Stimuli and Procedures}

The current study adopted the same set of stimulus used in reference (Vartanian et al., 2013), which had been frequently used to study aesthetic judgment of architectural space
(Vartanian et al., 2013, 2015, 2019; Coburn et al., 2020; Skov et al., 2021). The set of stimulus comprised 200 photos of architectural spaces of different styles. Each photo was categorized by three factors, i.e., ceiling height, openness and contour. Each factor had two levels, i.e., high and low for ceiling, open and enclosed for openness, curvilinear and rectilinear for contour. The categorization of each photo was confirmed by two researchers in the field of architectural aesthetics, i.e., two of the authors of reference (Vartanian et al., 2013). The combination of the factors at the various levels generated eight sets of images of spaces with different styles, and each set contained 25 photos (Figure 1).

The experiments were performed in a soundproof room. The participants sat in a chair and rest his or her chin on a chin rest. The height of the chair was adjusted to ensure that the line of sight was at approximately the upper quarter of the screen of the monitor. The experiment stimuli were presented on a 23inch monitor (Dell E2316H, resolution: $1920 \times 1080$, refresh rate: $60 \mathrm{~Hz}$, size: $509 \times 286 \mathrm{~mm}$ ). The eye distance from the screen was approximately $900 \mathrm{~mm}$. The view angle of the images were roughly $14 \times 9$ degree.

The study consisted of two conditions. Each condition included 200 trials, and 1 image was presented in each trial. The steps for each trial were as follows: First, a fixation cross was presented at the center of the screen for $1000 \mathrm{~ms}$. Then, a randomly selected stimulus image was presented for $200 \mathrm{~ms}$, which was followed by two questions asking the participant to score the image in terms of pleasantness and beauty $(1=$ very unpleasant/ugly; 5 = very pleasant/beautiful). One condition presented the original color images in reference (Vartanian et al., 2013), and the other condition presented the grayscale version of the images. Each RGB color image was converted into a grayscale image by averaging the three color channels (Figure 2).

\section{RESULTS}

\section{Aesthetic Judgment Under Short Viewing Time}

In the color-image condition, a three-way repeated measures ANOVA analysis (ceiling height $\times$ openness $\times$ contour) showed that the main effects of the three factors, i.e., ceiling height, openness and contour, were all statistically significant (Table 1). Overall, structures with high ceilings, open space or curvilinear contours were rated as being more pleasant and beautiful (Figure 3). These results were consistent with previous studies that employed 3,000-ms viewing time (Vartanian et al., 2013, 2015, 2019; Coburn et al., 2020; Skov et al., 2021).

\section{Aesthetic Judgment of Grayscale Images}

The grayscale-image condition was the same as the colorimage condition, except that the images were converted into grayscale. A three-way repeated measures ANOVA analysis (ceiling height $\times$ openness $\times$ contour) showed that the main effects of the three factors, i.e., ceiling height, openness and contour, were statistically significant (Table 1). Structures with high ceilings, open space, or curvilinear contours generated a higher level of pleasantness in the viewer than images 


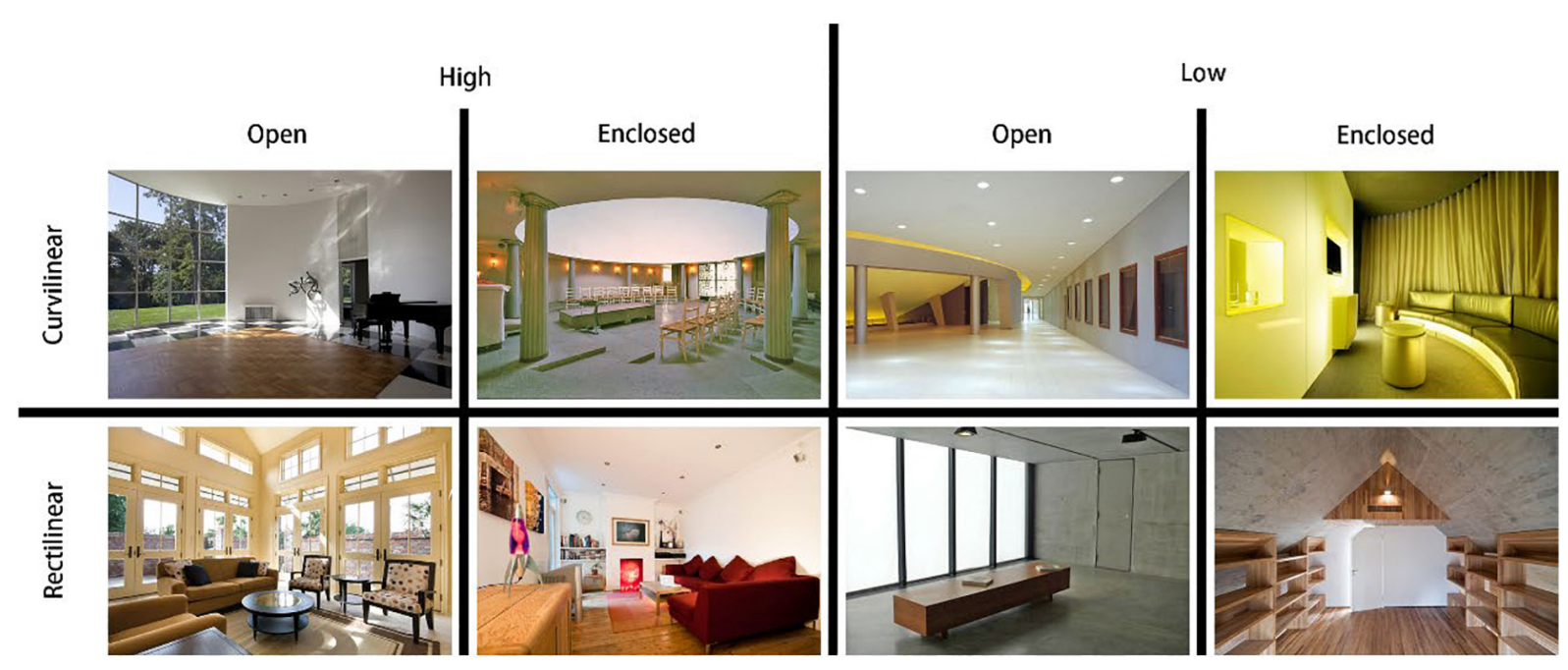

FIGURE 1 | Example of the stimulus. A total of 200 pictures were divided into 8 groups with 25 pictures in each group.

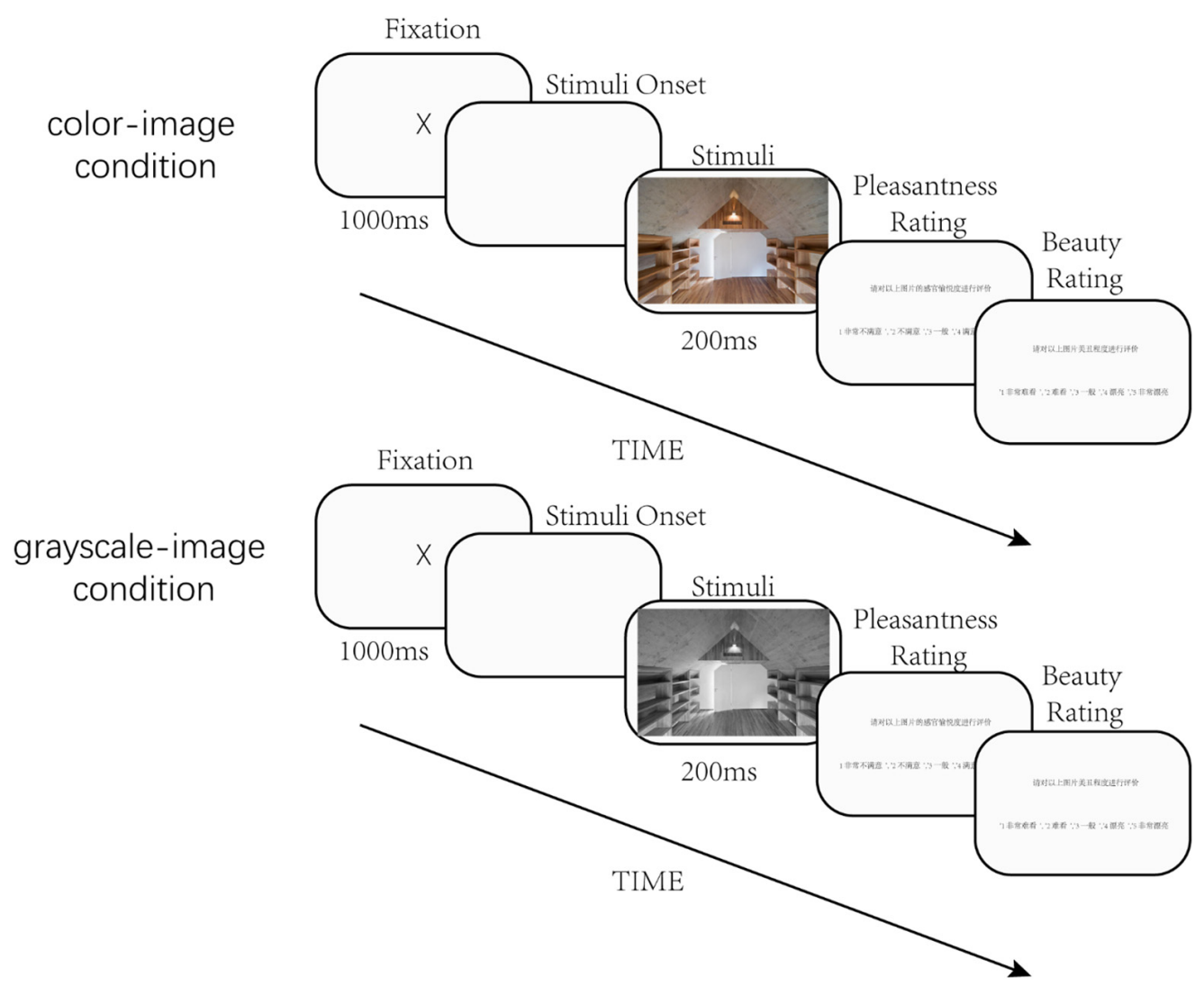

FIGURE 2 | |llustration of the experimental procedure.

of structures with low ceilings, enclosed space, or rectilinear contours (Figure 4). The results in the grayscale-image condition was consistent with the results in the color-image condition, suggesting that color does not significant affected aesthetic judgment of architectural space.

\section{DISCUSSION}

In this study, we find that when viewing images of architectural space for only $200 \mathrm{~ms}$, observers prefer space with higher ceilings, higher degree of openness, and curvilinear contours, compared 
TABLE 1 | ANOVA analyses of the beauty ratings for the short viewing time condition and the grayscale condition.

\begin{tabular}{|c|c|c|c|c|}
\hline \multirow[t]{2}{*}{ Factors } & \multicolumn{2}{|c|}{ Color condition } & \multicolumn{2}{|c|}{ Grayscale condition } \\
\hline & Pleasure $P(F)$ & Beauty $P(F)$ & Pleasure $P(F)$ & Beauty $P(F)$ \\
\hline Ceiling height & $<0.001(21.39)^{\star}$ & $<0.001(17.95)^{\star}$ & $<0.001(50.51)^{\star}$ & $<0.001(48.83)^{*}$ \\
\hline Degree of openness & $<0.001(75.11)^{\star}$ & $<0.001(62.18)^{\star}$ & $<0.001(81.39)^{\star}$ & $<0.001(69.31)^{\star}$ \\
\hline Contour type & $0.009(8.32)^{*}$ & $<0.001(20.25)^{\star}$ & $0.018(6.64)^{\star}$ & $<0.001(16.97)^{\star}$ \\
\hline Ceiling height $\times$ Degree of openness & $0.639(0.23)$ & $0.689(0.17)$ & $0.757(0.1)$ & $0.627(0.24)$ \\
\hline Ceiling height $\times$ Contour type & $0.11(2.8)$ & $0.023(6.01)^{*}$ & $<0.001(32.4)^{\star}$ & $<0.001(26.53)^{*}$ \\
\hline Degree of openness $\times$ Contour type & $<0.001(27.15)^{\star}$ & $<0.001(21.15)^{\star}$ & $<0.001(23.36)^{\star}$ & $<0.001(27.45)^{\star}$ \\
\hline Ceiling height $\times$ Degree of openness $\times$ Contour type & $0.035(5.14)^{\star}$ & $<0.001(23.26)^{\star}$ & $<0.001(19.78)^{\star}$ & $<0.001(40.12)^{*}$ \\
\hline
\end{tabular}

${ }^{*} p<0.05$. The main effect of each factor is significant, and the rating is higher for higher ceilings, more open space, and curvilinear contours.
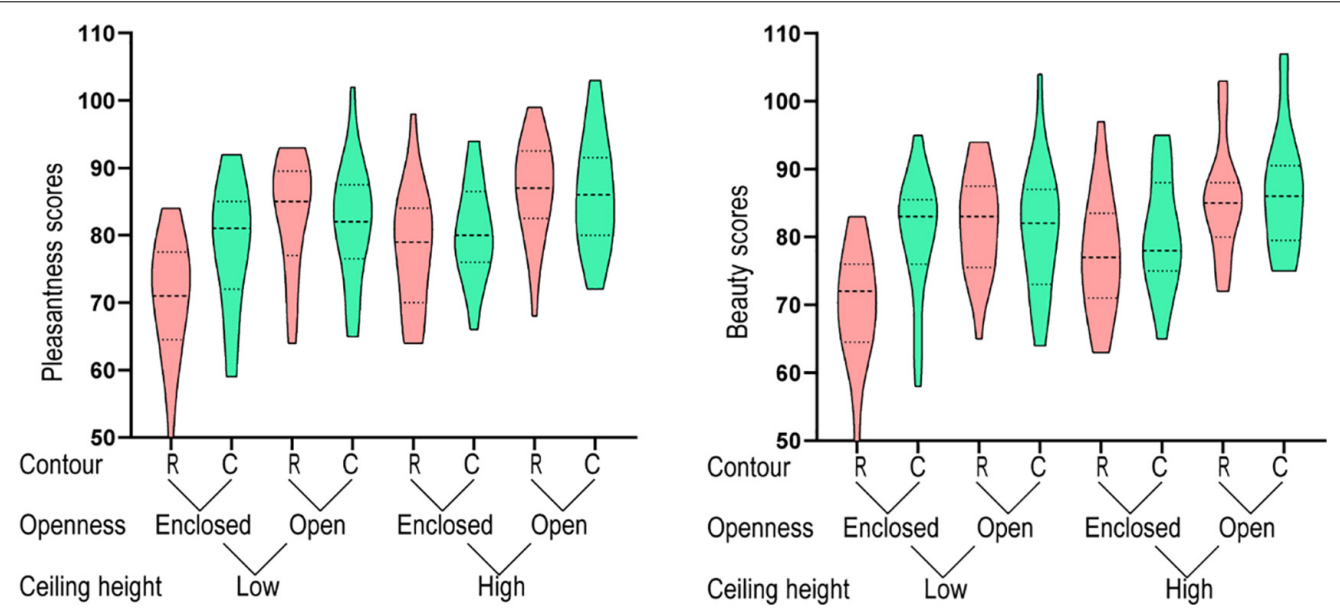

FIGURE 3 | Results for the color-image condition.
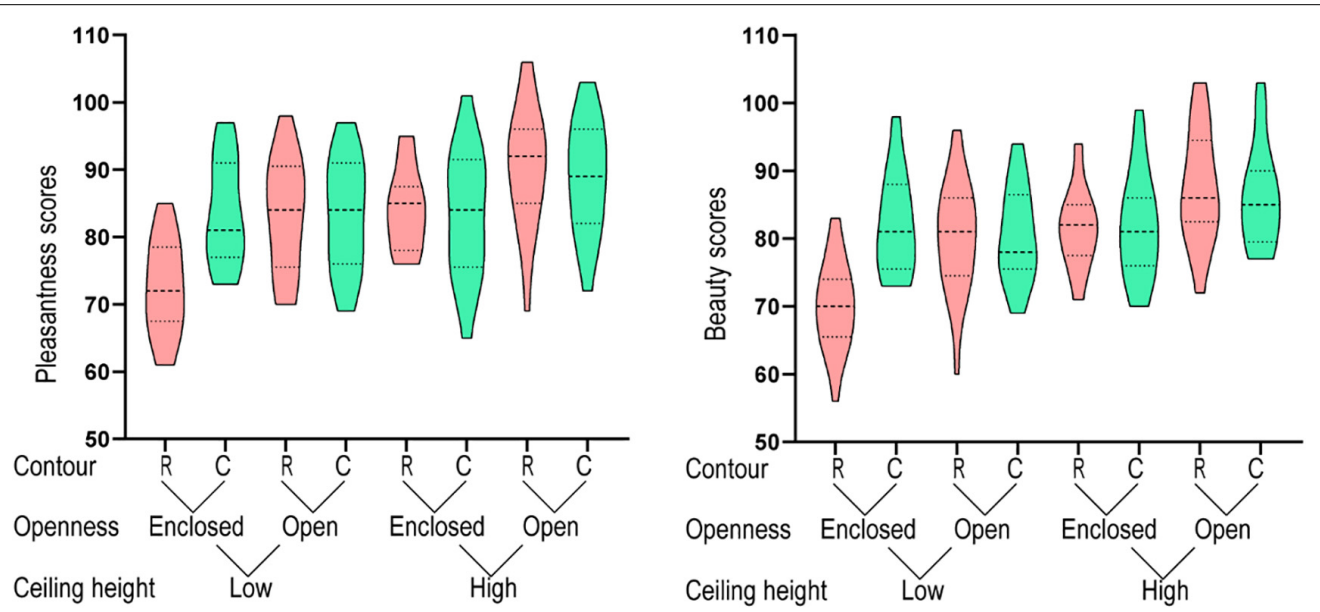

FIGURE 4 | Results for the grayscale-image condition.

with space with lower ceilings, lower degree of openness, and rectilinear contours. These conclusions hold for both color and grayscale images of architectural space, and are consistent with previous studies in which aesthetic ratings were collected for images presented for a much longer time, i.e., $3000 \mathrm{~ms}$.

Viewing time can strongly influence perception (Fei-Fei et al., 2007; Mullin et al., 2017). Furthermore, previous study shows that participants recognize normal color images faster than grayscale images (Oliva and Schyns, 2000), indicating that color plays an important role in the early stages of visual processing (Spence et al., 2006) and aids the human cognitive system in rapid recognition (Gegenfurtner and Rieger, 2000; Castelhano and Henderson, 2008). Color, however, is a feature that can be dissociated from architectural features such as ceiling height, 
openness, and contour, and therefore it is important to test whether the conclusions of previous studies on color images can generalize to grayscale images. Previous studies using color images have shown that observers prefer high ceilings, open space, and curvilinear contours (Vartanian et al., 2013, 2015, 2019; Coburn et al., 2020; Skov et al., 2021), and the current study show that observers prefer the same set of features when viewing grayscale images.

In sum, here we demonstrated that ceiling height, openness, and contour can reliably influence the viewers' aesthetic judgment, despite of changes in the stimulus duration and color. The results suggest that future studies can collect aesthetic ratings of architectural spaces in a more effective way.

\section{DATA AVAILABILITY STATEMENT}

The raw data supporting the conclusions of this article will be made available by the authors, without undue reservation.

\section{REFERENCES}

Bar, M., and Neta, M. (2006). Humans prefer curved visual objects. Psychol. Sci. 17, 645-648. doi: 10.1111/j.1467-9280.2006.01759.x

Berlyne, D. E. (1973). Aesthetics and psychobiology. J. Aesthetics Art Criticism 31:553. doi: $10.2307 / 429334$

Castelhano, M. S., and Henderson, J. M. (2008). The influence of color on the perception of scene gist. J. Exp. Psychol. Hum. Percept. Perform. 34, 660-675. doi: 10.1037/0096-1523.34.3.660

Coburn, A., Vartanian, O., Kenett, Y. N., Nadal, M., Hartung, F., HaynLeichsenring, G., et al. (2020). Psychological and neural responses to architectural interiors. Cortex 126, 217-241. doi: 10.1016/j.cortex.2020.01.009

Dazkir, S. S., and Read, M. A. (2011). Furniture forms and their influence on our emotional responses toward interior environments. Environ. Behav. 44, $722-732$.

Fei-Fei, L., Iyer, A., Koch, C., and Perona, P. (2007). What do we perceive in a glance of a real-world scene? J. Vis. 7:10. doi: 10.1167/7.1.10

Franz, G., von der Heyde, M., and Bülthoff, H. H. (2005). An empirical approach to the experience of architectural space in virtual reality-exploring relations between features and affective appraisals of rectangular indoor spaces. Autom. Constr. 14, 165-172.

Gegenfurtner, K. R., and Rieger, J. (2000). Sensory and cognitive contributions of color to the recognition of natural scenes. Curr. Biol. 10, 805-808. doi: $10.1016 / \mathrm{s} 0960-9822(00) 00563-7$

Jacobsen, T. (2006). Bridging the arts and sciences: a framework for the psychology of aesthetics. Leonardo 39, 155-162.

Leder, H., and Carbon, C. (2005). Dimensions in appreciation of car interior design. Appl. Cogn. Psychol. 19, 603-618.

Meyers-Levy, J., Zhu, R. J., and John, D. S. A. E. (2007). The influence of ceiling height: the effect of priming on the type of processing that people use. J. Consum. Res. 34, 174-186. doi: 10.1086/519146

Mullin, C., Hayn-Leichsenring, G., Redies, C., and Wagemans, J. (2017). The gist of beauty: an investigation of aesthetic perception in rapidly presented images. Electronic Imaging 2017, 248-256. doi: 10.2352/issn.2470-1173.2017.14.hvei152

Oliva, A., and Schyns, P. G. (2000). Diagnostic colors mediate scene recognition. Cogn. Psychol. 41, 176-210. doi: 10.1006/cogp.1999.0728

Skov, M., Vartanian, O., Navarrete, G., Modroño, C., Chatterjee, A., Leder, H., et al. (2021). Differences in regional grey matter volume predict the extent to which openness influences judgments of beauty and pleasantness of interior architectural spaces. Ann. N. Y. Acad. Sci. 2021:13. doi: 10.1111/nyas.14684

\section{ETHICS STATEMENT}

The studies involving human participants were reviewed and approved by Research Ethics Committee of Zhejiang University School of Medicine. The patients/participants provided their written informed consent to participate in this study.

\section{AUTHOR CONTRIBUTIONS}

$\mathrm{AD}$ and $\mathrm{HF}$ conceived the experiments and wrote the manuscript. $\mathrm{AD}$ and JY performed the experiments. JW analyzed the data. All authors edited the manuscript.

\section{SUPPLEMENTARY MATERIAL}

The Supplementary Material for this article can be found online at: https://www.frontiersin.org/articles/10.3389/fpsyg. 2021.752996/full\#supplementary-material

Spence, I., Wong, P., Rusan, M., and Rastegar, N. (2006). How color enhances visual memory for natural scenes. Psychol. Sci. 17, 1-6. doi: 10.1111/j.1467-9280.2005. 01656.x

Stamps, A. (2011). Effects of area, height, elongation, and color on perceived spaciousness. Environ. Behav. 2, 252-273. doi: 10.1177/0013916509354696

Stamps, A. E. (2010). Effects of permeability on perceived enclosure and spaciousness. Environ. Behav. 6, 864-886. doi: 10.1177/0013916509337287

Sternberg, E. M., and Wilson, M. A. (2006). Neuroscience and architecture: seeking common ground. Cell 127, 239-242. doi: 10.1016/j.cell.2006.10.012

Vartanian, O., Navarrete, G., Chatterjee, A., Fich, L. B., Gonzalez-Mora, J. L., Leder, H., et al. (2015). Architectural design and the brain: effects of ceiling height and perceived enclosure on beauty judgments and approach-avoidance decisions. J. Environ. Psychol. 41, 10-18. doi: 10.1016/j.jenvp.2014.11.006

Vartanian, O., Navarrete, G., Chatterjee, A., Fich, L. B., Leder, H., Modroño, C., et al. (2013). Impact of contour on aesthetic judgments and approachavoidance decisions in architecture. Proc. Natl. Acad. Sci. U.S.A. 110(Suppl. 2), 10446-10453. doi: 10.1073/pnas.1301227110

Vartanian, O., Navarrete, G., Chatterjee, A., Fich, L. B., Leder, H., Modroño, C., et al. (2019). Preference for curvilinear contour in interior architectural spaces: evidence from experts and nonexperts. Psychol. Aesthetics Creativ. Arts 13, 110-116. doi: 10.1037/aca0000150

Weber, R., Schnier, J., and Jacobsen, T. (2008). Aesthetics of streetscapes: influence of fundamental properties on aesthetic judgments of urban space. Percept. Motor Skills 106, 128-146. doi: 10.2466/pms.106.1.128-146

Conflict of Interest: The authors declare that the research was conducted in the absence of any commercial or financial relationships that could be construed as a potential conflict of interest.

Publisher's Note: All claims expressed in this article are solely those of the authors and do not necessarily represent those of their affiliated organizations, or those of the publisher, the editors and the reviewers. Any product that may be evaluated in this article, or claim that may be made by its manufacturer, is not guaranteed or endorsed by the publisher.

Copyright (c) 2022 Dai, Wang, Yu and Fukuda. This is an open-access article distributed under the terms of the Creative Commons Attribution License (CC BY). The use, distribution or reproduction in other forums is permitted, provided the original author(s) and the copyright owner(s) are credited and that the original publication in this journal is cited, in accordance with accepted academic practice. No use, distribution or reproduction is permitted which does not comply with these terms. 